\title{
CHECKLIST DAS MACRÓFITAS AQUÁTICAS DO PANTANAL, BRASIL $^{1}$
}

\author{
Vali Joana Pott ${ }^{2}$ \\ Arnildo Pott ${ }^{2}$
}

Recebido em 31/10/96. Aceito em 31/12/97

\begin{abstract}
RESUMO - (Checklist das macrófitas aquáticas do Pantanal, Brasil). O Pantanal, por ser alagável, é ambiente favorável ao desenvolvimento de muitas plantas aquáticas. Para atender demandas de levantamentos florísticos regionais, fez-se a listagem das macrófitas aquáticas do Pantanal, com base em coletas depositadas nos Herbários CPAP(EMBRAPA), COR (Universidade Federal de Mato Grosso do Sul), $\mathrm{CH}$ (Universidade Federal de Mato Grosso) e K (Kew). Até o momento, foram levantadas 242 espécies, distribuidas em 106 gêneros e 54 familias. As familias mais numerosas são Poaceae (22 espécies), Cyperaceae(17), Leguminosae e Scrophulariaceae(14), Alismataceae e Onagraceae(13), Pontederiaceae(11), Lentibulariaceae(10), Characeae(9), Lemnaceae, Malvaceae e Nymphaeaceae(8). A maioria das demais famílias apresentam uma ou duas espécies cada. Quanto à forma biológica, $39 \%$ são plantas emergentes, $28 \%$ anfibias, $11,5 \%$ flutuantes fixas, $8 \%$ flutuantes livres, $8 \%$ submersas fixas, $3,8 \%$ submersas livres e 1,7\% epifitas. Os principais gêneros em número de espécies são Nymphaea, Utricularia, Echinodorus, Ludwigia, Polygonum, Aeschynomene, Cyperus, Eleocharis e Bacopa.
\end{abstract}

Palavras-chave: plantas aquáticas, hidrófito, área úmida, flora, Pantanal

\begin{abstract}
Checklist of the aquatic macrophytes of the Pantanal, Brazil). The Pantanal, being a wetland, is a favorable environment for the development of many aquatic plants. This list of aquatic macrophytes of the Pantanal was made based on field collections, deposited at Herbarium CPAP (EMBRAPA), COR (Universidade Federal de Mato Grosso do Sul), CH (Universidade Federal de Mato Grosso) and K (Kew), to support regional floristic surveys. Until present, 242 species were listed, distributed within 106 genera and 54 families. The most numerous families were Poaceae ( 22 species) Cyperaceae(17), Leguminosae and Scrophulariaceae(14), Alismataceae and Onagraceae(13), Pontederiaceae (11), Lentibulariaceae(10), Characeae(9), Lemnaceae, Malvaceae and Nymphaeaceae(8). The majority of other families has one or two species each. The life form spectrum includes $39 \%$ emergent plants, $28 \%$ amphibious, $11,5 \%$ rooted floating, $8 \%$ free floating, $8 \%$ rooted submerged, $3,8 \%$ free submerged and $1,7 \%$ epiphytes. The most important genera in number of species are Nymphaea, Utricularia, Echinodorus, Ludwigia, Polygonum, Aeschynomene, Cyperus, Eleocharis and Bacopa.
\end{abstract}

Key words: aquatic plants, hydrophytes, wetland, flora, Pantanal

\footnotetext{
Trabalho apresentado no III Encontro de Botânicos do Centro Oeste, Cuiabá, 1995; Auxilios PCBAP-PNMA e CECITEC-MS

Centro de Pesquisa Agropecuária do Pantanal-EMBRAPA, C.P. 109 (Herbário), CEP 79320-900, Corumbá, MS, Brasil
} 


\section{Introdução}

O Pantanal, por ser alagável, é uma área favorável ao desenvolvimento de muitas plantas aquáticas. Os ambientes são os mais diversos, como rios, corixos, vazantes, baías (lagoas), lagoas de meandro, brejos, campos alagáveis e caixas de empréstimo. As salinas (lagoas alcalinas) geralmente não apresentam macrófitas, apenas algas.

Macrófitas são importantes na cadeia trófica de ecossistemas aquáticos, porque fornecem abrigo a peixes, insetos aquáticos, moluscos e também ao perifiton. Fazem autodepuração das águas pela assimilação de nutrientes e retenção de sedimentos (Hamilton 1993). Comunidades características são os camalotes e baceros ou ilhas flutuantes, que se deslocam rio abaixo por ocasião da enchente.

$\mathrm{O}$ conceito de planta aquática é muito discutido e varia de acordo com os autores. Para o International Program of Biology (IBP), "macrófita aquática" é a denominação genérica (independente de aspectos taxônomicos) mais adequada para vegetais que habitam desde brejos até ambientes verdadeiramente aquáticos (Esteves 1988). Segundo Fasset (1966) e Cook (1974), engloba todas as plantas cujas partes fotossinteticamente ativas estão permanentemente, ou por alguns meses, ou cada ano, a submersas ou flutuantes em água e sejam visíveis a olho nu, englobando Charophyta, Bryophyta, Pteridophyta e Spermatophyta. Conceito semelhante é dado por Irgang \& Gastal (1996), acrescentando "em água doce ou salobra". Para Martins \& Carauta (1984), são todas as plantas que vivem na água ou sobre ela, num sentido amplo e subjetivo, e o termo ecológico correspondente é hidrófito. Porém, segundo Esteves (1988), o termo "macrófitas aquáticas" é amplamente utilizado em todo o mundo e já está incorporado à literatura científica.

São escassas as informações sobre plantas de lagoas e de áreas alagadas do Pantanal. Hoehne $(1923 ; 1948)$ possivelmente tenha sido quem mais escreveu sobre plantas aquáticas da flora pantaneira. Silva (1984) fez um estudo sobre a composição florística do camalote ou batume em Mato Grosso. Conceição \& Paula (1986) citam algumas plantas, distinguindo ambiente inundado e inundável. Allem \& Valls (1987) fizeram levantamento das gramíneas e leguminosas aquáticas na região. Pott et al. (1989) realizaram estudo quantitativo de uma lagoa da fazenda Nhumirim. Ainda, existe outro estudo quantitativo das comunidades da flora aquática de três lagoas da fazenda Leque, sub-região do Abobral (Pott et al. 1992). Prado et al.(1994) fizeram um estudo da sucessão vegetal de uma área alagável na sub-região de Poconé, apresentando lista florística de 48 espécies registradas em parcelas, além de outras daquele local, quase todas coincidentes com as da presente listagem. Recentemente, Schessl (1997) estudou a florística e fitossociologia de quatro áreas no Pantanal de Poconé, MT, onde 118 espécies coincidem com as 242 espécies listadas neste trabalho. De poucas famílias há revisão ou levantamento taxonômico detalhado, como Lemnaceae (Pott 1993) e Characeae (Bueno 1993).

Para atender demandas por levantamentos florísticos regionais, fez-se a listagem das macrófitas do Pantanal brasileiro, com base em coletas realizadas nos últimos 10 anos.

\section{Material e métodos}

O Pantanal situa-se entre as coordenadas $16^{\circ}$ e $22^{\circ} \mathrm{S}$, e $55^{\circ}$ e $58^{\circ} \mathrm{W}$. É dividido em 11 sub-regiões, segundo Silva et al. (1995). Possui área de $140.000 \mathrm{~km}^{2}$, dos quais 
mais da metade são cobertos por água na época da cheia. O clima é do tipo Aw segundo Koeppen, com precipitação anual em torno de $1.100 \mathrm{~mm}$.

A listagem foi feita com base em coletas dos autores entre os anos 1986 e 1996, perfazendo o total de 1900 espécimes, com alguns acréscimos de outros coletores. As coletas botânicas abrangem todas as sub-regiões do Pantanal e estão depositadas no Herbário CPAP, do Centro de Pesquisa Agropecuária do Pantanal, EMBRAPA (Empresa Brasileira de Pesquisa Agropecuária), Corumbá, que possui acervo representativo da região. Foram consultados também os Herbários COR da UFMS (Universidade Federal de Mato Grosso do Sul), CH da UFMT (Universidade Federal do Mato Grosso) e K (Royal Botanic Gardens, Kew, Inglaterra). A lista florística contém as familias e os gêneros em ordem alfabética, os nomes populares da região e as formas biológicas, seguidos das iniciais dos coletores e número de coleta. O sistema de classificação usado foi o de Cronquist (1981) para Magnoliophyta (Angiospermas), exceto Leguminosae; o dicionário de Willis (1973) para Pteridophyta, e Crandall-Stotler (1980) para Hepatophyta.

As formas biológicas foram consideradas de acordo com Irgang et al. (1984); a forma epifita, segundo Tur (1965), e as Characeae, conforme Bueno (1993). Para os cálculos de porcentagem das formas biológicas foi considerada a forma predominante de cada espécie de acordo com o ambiente (excluiram-se Myriophylum matogrossense e Ottelia brasiliensis). Incluiram-se plantas de lugares úmidos na categoria de anfíbias.

\section{Resultados e discussão}

Foram levantadas 242 espécies, distribuídas em 106 gêneros e 54 famílias; destas, 22 espécies são representadas por Criptógamas, sendo 9 Charophyta, 1 Hepatophyta e 12 Pteridophyta (Tab. 1). As famílias mais numerosas são Poaceae (22 espécies), Cyperaceae(17), Leguminosae e Scrophulariaceae(14), Alismataceae e Onagraceae(13), Pontederiaceae(11), Lentibulariaceae(10), Characeae(9), Lemnaceae, Malvaceae e Nymphaeaceae(8); as famílias com 2 espécies cada são Azollaceae, Marsileaceae, Parkeriaceae, Acanthaceae, Amaranthaceae, Apiaceae, Cabombaceae, Ceratophyllaceae, Commelinaceae, Marantaceae, Melastomataceae, Menyanthaceae e Najadaceae; enquanto Ricciaceae, Equisetaceae, Hemionitidaceae, Isoetaceae, Apocynaceae, Cannaceae, Cucurbitaceae, Gentianaceae, Haloragaceae, Hydrophyllaceae, Mayacaceae, Potamogetonaceae, Solanaceae, Sphenocleaceae e Typhaceae têm apenas uma espécie. A Fig. 1 mostra as famílias com maior número de espécies.

As formas biológicas emergentes (E) e anfibias (A) são as mais representadas ( $39 \%$ e $28 \%$, respectivamente), seguidas por flutuantes fixas (FF) $(11,5 \%)$, flutuantes livres (FL) (8\%), submersas fixas (SF) (8\%), submersas livres (SL) $(3,8 \%)$, e epifitas (EP) com apenas 4 espécies $(1,7 \%$ ) (Tab. 2). As famílias e gêneros predominantes em cada forma biológica são:

E - Poaceae, com vários gêneros, e Alismataceae, com Echinodorus.

A - Scrophulariaceae, com Bacopa, e Onagraceae, com Ludwigia.

FF - Nymphaeaceae, com Nymphaea.

FL - Lemnaceae, com seus 4 gêneros.

SF - Characeae, com Chara e Nitella.

$\mathrm{Sl}$ - Lentibulariaceae, com Utriclaria.

EP - Cyperaceae, com Oxycaryum (em grande área ocupada pelo bacero) além de Orchidaceae, com 2 espécies. 
Tabela 1. Lista das macrófitas aquáticas do Pantanal, em ordem alfabética de familia e gênero, nome popular (se houver), e forma biológica : $\mathrm{FL}=$ Flutuante livre; $\mathrm{FF}=\mathrm{Flutuante}$ fixa; $\mathrm{SL}=$ Submersa livre; $\mathrm{SF}=\mathrm{Submersa}$ fixa; $\mathrm{A}=$ Anfibia; $\mathrm{E}=$ Emergente; $\mathrm{EP}=$ Epifita. Nomes abreviados dos coletores e número: $\mathrm{ACA}=$ Antônio $\mathrm{C}$. Allem; $\mathrm{ACC}=$ Armando $\mathrm{C}$. Cervi; $\mathrm{AP}=$ Arnildo Pott; $\mathrm{CJAF}=$ Celso João A. Ferreira; $\mathrm{CDKC}=$ Christopher D. K. Cook; $\mathrm{GH}=$ Gerdt Hatschbach; $\mathrm{NCB}=$ Norma C. Bueno; $\mathrm{ODB}=$ Oslain D. Branco; SH=Stefen Hamilton; VJP=Vali Joana Pott; CH (UFMT) = Herbário Central, Universidade Federal do Mato Grosso; CPAP=Herbário CPAP.

\begin{tabular}{lccc}
\hline Familia/Espécie & $\begin{array}{c}\text { Nome } \\
\text { popular }\end{array}$ & $\begin{array}{c}\text { Forma } \\
\text { biol. }\end{array}$ & $\begin{array}{c}\text { Número } \\
\text { coletor }\end{array}$ \\
\hline
\end{tabular}

\section{CHARACEAE - Charophyta}

Chara fibrosa C. Agardh ex Bruzelius emend. R.D. Wood

C. guairensis $\mathrm{R}$. Bicudo

C. rusbyana Howe

Nitella acuminata A. Braun

N. cernua A. Braun

N. furcata (Roxb. ex Bruzelius) C. Agardh emend. R.D. Wood

N. gollmeriana A. Braun

N. subglomerata A. Braun

N. transluscens (Pers.) C. Agardh emend. R.D. Wood

RICCIACEAE - Hepatophyta (Bryophyta)

Ricciocarpus natans (L.) Corda

HEMIONITIDACEAE - Pteridophyta

Pityrogramma calomelanos (L.) Link var. calomelanos

\section{AZOLLACEAE - Pteridophyta}

Azolla caroliniana Willd.

A. filiculoides Lam.

EQUISETACEAE

Equisetum giganteum $\mathrm{L}$.

ISOETACEAE - Pteridophyta

Isoetes pedersenii Hickey

MARSILEACEAE - Pteridophyta

Marsilea crotophora D. M. Johnson

M. deflexa A. Braun

PARKERIACEAE - Pteridophyta

Ceratopteris pteridoides (Hook.) Hieron.

C. thalictroides (L.) Brongn.

\section{SALVINIACEAE - Pteridophyta}

Salvinia auriculata Aubl.

S. biloba Raddi emend de la Sota

$S$. minima Baker

ACANTHACEAE - Magnoliophyta (Angiosp.)

Hygrophila guyanensis Nees

Justicia laevilinguis (Nees) Lindau

\section{ALISMATACEAE}

Echinodorus bolivianus (Rusby) Holm-Niels.

Echinodorus cordifolius (L.) Griseb.

E. grandiflorus (Cham. \& Schltdl.) Micheli ssp. grandiflorus

E. lanceolatus Rataj

E. longipetalus Micheli

E. macrophyllus ssp. scaber (Rataj) Haynes \& Holm-Nielsen

E. paniculatus Micheli

Echinodorus subulatus (Mart.) Griseb.

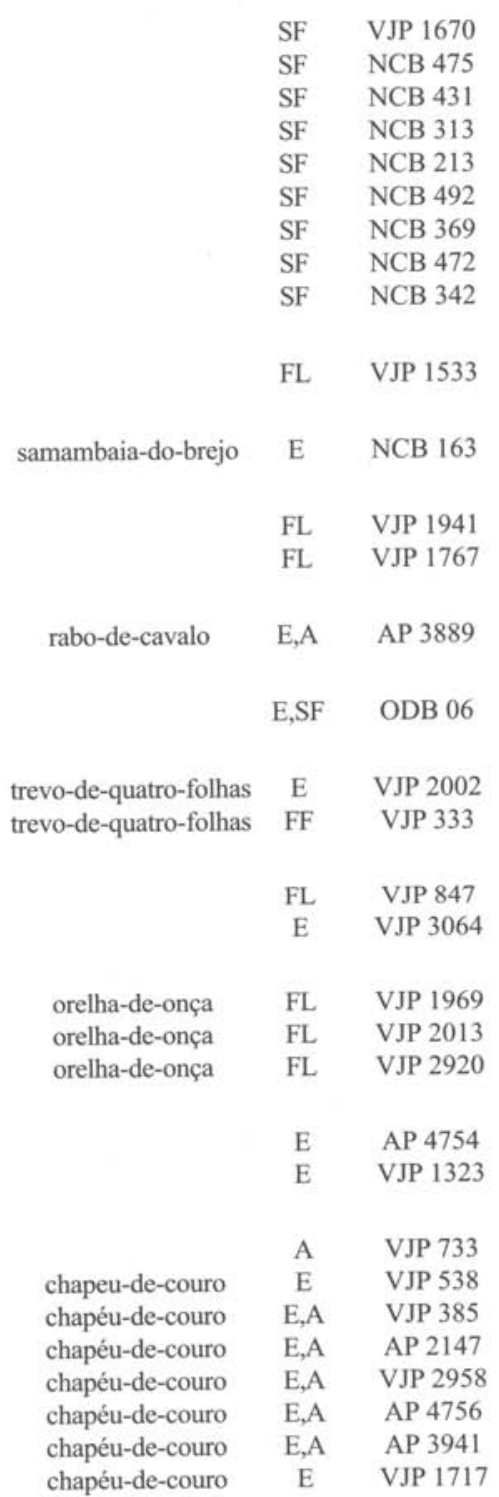


Familia/Espécie

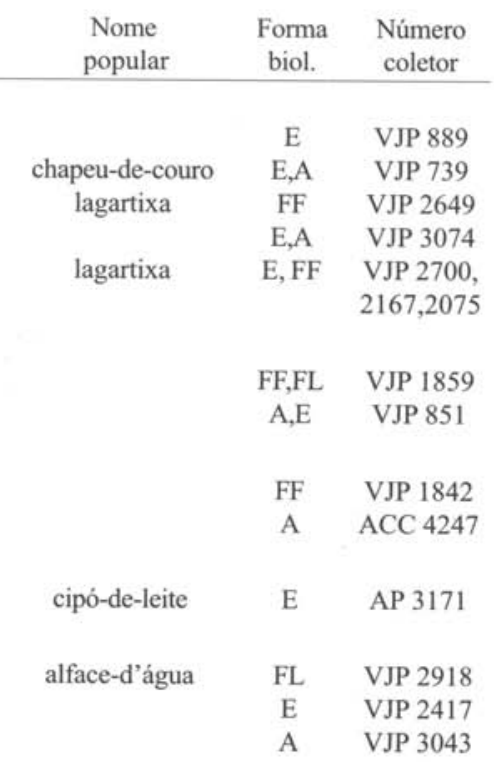

E. tenellus (Mart.) Buchenau

E. teretoscapus R.R.Haynes \& Holm-Niels.

Sagittaria guayanensis H.B.K.

S. montevidensis Cham. \& Schltdl.

S. rhombifolia Cham.

\section{AMARANTHACEAE}

Alternanthera aquatica (Parodi) Chodat

A. philoxeroides (Mart.) Griseb.

APIACEAE (Umbelliferae)

Hydrocotyle ranunculoides L. f.

Hydrocotyle verticillata Thunb.

\section{APOCYNACEAE}

Rhabdadenia pohlii Müll. Arg.

\section{ARACEAE}

Pistia stratiotes $\mathrm{L}$.

Urospatha sagittifolia (Rudge) Schott

Xanthosoma striatipes (Kunth \& Bouché) Mad.

ASTERACEAE (Compositae)

Aspilia latissima Malme

Eclipta prostrata (L.) L. (= Eclipta alba (L.) Hassk.)

mirassol; fumeiro

A $\quad$ AP 3919

Enidra radicans (Willd.) Lack ( $=$ E. anagallis Gardner)

Gymnocoronis spilanthoides (Don) DC.

Mikania micrantha H.B.K. (= M. scandens Willd.)

Pacourina edulis Aubl.

jasmim-do-campo

A $\quad$ GH 60939

A VJP 1841

E $\quad$ AP 6238

CABOMBACEAE

Cabomba furcata Schult. \& Schult. f.

( $=$ C. piauhyensis Gardner)

C. caroliniana A. Gray

CANNACEAE

Canna glauca $\mathrm{L}$.

\section{CERATOPHYLLACEAE}

Ceratophyllum submersum (Gray)

Wilmot-Dear var. echinatum Gray

C. demersum $\mathrm{L}$.

\section{COMMELINACEAE}

Commelina schomburgkiana Klotzsch ex Seub.

Murdannia sp.

\section{CONVOLVULACEAE}

Ipomoea alba $\mathrm{L}$.

I. asarifolia (Desr.) Roem. \& Schult.

I. carnea ssp. fistulosa (Mart. ex Choisy) D.F. Austin

I. rubens Choisy

E,A $\quad$ VJP 878

E VJP 2520

$\begin{array}{lll}\text { lodo } & \text { SF } & \text { VJP } 844 \\ \text { lodo } & \text { SF } & \text { VJP 1826 }\end{array}$

cana-do-brejo; $\quad$ E $\quad$ NCB 209

banana-d'água

$\begin{array}{lll}\text { lodo } & \text { SL } & \text { VJP } 2526 \\ \text { lodo } & \text { SL } & \text { VJP } 2539\end{array}$

$\begin{array}{lll}\text { santa-luzia } & \text { E } & \text { VJP } 3191 \\ & \text { E } & \text { VJP 595 }\end{array}$

viuviu; $\quad$ A $\quad$ VJP 1396

abre-noite-fecha-dia

$\begin{array}{lll} & \text { A } & \text { VJP 3034 } \\ \text { algodão-bravo } & \text { A } & \text { NCB 28 } \\ & \text { A } & \text { VJP 854 }\end{array}$

\section{CUCURBITACEAE}

Cyclanthera hystrix (Gill.) Arn.

E $\quad$ AP 6224 


\section{Familia/Espécie}

Nome

popular

\section{CYPERACEAE}

Cladium jamaicense $\mathrm{Crtz}$.

Cyperus corymbosus v. subnodosus (Nees \& Meyen) Kük.

C. esculentus L. var. leptostachyus Boeck.

C. giganteus Vahl

C. odoratus $\mathrm{L}$.

C. prolixus H.B.K.

Eleocharis acutangula (Roxb.) Steud.

E. barrosoi Svens.

E. elegans (H.B.K.) Roem. \& Schult.

E. geniculata (L.) Roem. \& Schult.

E. interstincta (Vahl) Roem. \& Schult.

E. minima Kunth

E. nudipes (Kunth.) Palla

Fuirena umbellata Rottb.

Oxycaryum cubense (Poepp. \& Kunth) Lye

(= Scirpus cubensis Poepp. \& Kunth)

Rhynchospora velutina (Kunth.) Boeck.

Scleria leucocephala (= S. pterota Presl)

\section{EUPHORBIACEAE}

Caperonia castaneifolia (L.) A.St.-Hil.

C. palustris (L.) A. St.-Hil.

Phyllanthus fluitans Müll. Arg.

P. lindbergii Müll. Arg.

\section{GENTIANACEAE}

Curtia tenuifolia (Aubl.) Knobl.

HALORAGACEAE

${ }^{* *}$ Myriophyllum matogrossense Hoehne

\section{HYDROCHARITACEAE}

Egeria najas Planch.

Limnobium laevigatum (Humb. \& Bonpl. ex Willd.)

Heine (=L. stoloniferum Griseb.)

**Ottelia brasiliensis (Planch.) Walpers

\section{HYDROPHYLLACEAE}

Hydrolea spinosa $\mathrm{L}$.

\section{LAMIACEAE (Labiatae)}

Hyptis lappacea Benth.

H. lorentziana $\mathrm{O}$. Hoffm.

H. microphylla Pohl ex Benth.

\section{LEGUMINOSAE}

Aeschynomene americana L.

A. ciliata Vog.

A. denticulata Rudd.

A. fluminensis Vell.

A. sensitiva $\mathrm{Sw}$.

Discolobium leptophyllum Benth.

D. psoraliaefolium Benth.

D. pulchellum Benth.

Neptunia prostrata (Lam.) Baill.

(=N. oleracea (Lour.) Baill.; N. natans (L.f.) Drude)

\begin{tabular}{|c|c|c|}
\hline \multirow[t]{3}{*}{ capim-navalha } & E & AP 3891 \\
\hline & A & VJP 1007 \\
\hline & A & VJP 1180 \\
\hline \multirow[t]{3}{*}{ pirizeiro } & $\mathrm{E}$ & VJP 1550 \\
\hline & A & AP 2664 \\
\hline & E & AP 3347 \\
\hline cebolinha & $\mathrm{E}$ & AP 3055 \\
\hline lodo & A & AP 3304 \\
\hline \multirow[t]{2}{*}{ cebolinha } & E & AP 3079 \\
\hline & $\mathrm{E}$ & AP 3487 \\
\hline cebolinha & $\mathrm{E}$ & AP 2134 \\
\hline \multirow[t]{2}{*}{ lodo } & A,SF & VJP 1038 \\
\hline & A & VJP 3159 \\
\hline \multirow{3}{*}{$\begin{array}{l}\text { capim-navalha } \\
\text { bacero }\end{array}$} & $\mathrm{E}$ & VJP 2052 \\
\hline & EP & VJP 2569 \\
\hline & A & VJP 3181 \\
\hline capim-navalha & $\mathrm{E}$ & VJP 3182 \\
\hline \multirow[t]{3}{*}{ erva-de-bicho-branca } & $\mathrm{A}, \mathrm{E}$ & AP 3741 \\
\hline & $\mathrm{E}$ & VJP 1853 \\
\hline & FL & VJP 1768 \\
\hline \multirow[t]{3}{*}{ corticinha } & $\mathrm{E}$ & AP 3100 \\
\hline & A & AP 2251 \\
\hline & SF & $\mathrm{s} / \mathrm{n}$ \\
\hline \multirow{3}{*}{$\begin{array}{l}\text { lodinho-branco } \\
\text { camalotinho }\end{array}$} & SF & VJP 587 \\
\hline & $\mathrm{FL}$ & VJP 846 \\
\hline & SF & $\mathrm{CH}, \mathrm{s} / \mathrm{n}$ \\
\hline \multirow[t]{7}{*}{ amoroso } & $\mathrm{A}, \mathrm{E}$ & VJP 1928 \\
\hline & A & VJP 2519 \\
\hline & A & AP 5086 \\
\hline & A & AP 5708 \\
\hline & $\mathrm{A}, \mathrm{E}$ & VJP 241 \\
\hline & $\mathrm{E}$ & VJP 1263 \\
\hline & E & AP 2691 \\
\hline cortiça & $\mathrm{A}, \mathrm{E}$ & VJP 1022 \\
\hline cortiça & $\mathrm{A}, \mathrm{E}$ & AP 1755 \\
\hline cortiça & $\mathrm{E}$ & CPAP 16060 \\
\hline cortiça & $\mathrm{A}, \mathrm{E}$ & AP 2575 \\
\hline cortiça & $\mathrm{E}, \mathrm{A}$ & AP 6226 \\
\hline drume-drume & $\mathrm{FF}, \mathrm{A}, \mathrm{E}$ & VJP 2080 \\
\hline
\end{tabular}


Familia/Espécie

$\begin{array}{ccc}\begin{array}{c}\text { Nome } \\ \text { popular }\end{array} & \begin{array}{c}\text { Forma } \\ \text { biol. }\end{array} & \begin{array}{c}\text { Número } \\ \text { coletor }\end{array} \\ \text { drume-drume } & \text { E,A } & \text { VJP 3204 } \\ & \text { A, } & \text { AP 4928 } \\ & \text { E,A } & \text { AP 6227 } \\ & \text { E } & \text { AP 2639 } \\ & \text { E } & \text { AP 5131 } \\ \text { açude } & \text { FL } & \text { VJP 2295 } \\ & \text { FL } & \text { VJP 2124 } \\ & \text { FL } & \text { VJP 2532 } \\ & \text { FL } & \text { VJP 2135 } \\ & \text { FL } & \text { VJP 2238 } \\ & \text { FL } & \text { VJP 2302 } \\ & \text { FL } & \text { VJP 1992 } \\ & \text { FL } & \text { VJP 2305 } \\ & & \\ & \text { SL } & \text { AP 2227 } \\ \text { lodo } & \text { SL } & \text { AP 6187 } \\ \text { lodo } & \text { SL } & \text { VJP 2570 } \\ \text { lodo } & \text { EP,SL } & \text { VJP 718 } \\ \text { lodo } & \text { SL } & \text { AP 2244 } \\ \text { lodo } & \text { SL } & \text { SH 05 } \\ & \text { SL } & \text { VJP 1483 } \\ & \text { A } & \text { AP 2915 } \\ & \text { A } & \text { VJP 2950 } \\ & \text { SL } & \text { VJP 1881 }\end{array}$

LEGUMINOSAE

N. plena (L.) Benth.

Senna pendula (Willd.) Irw. \& Barn.

Sesbania exasperata H.B.K.

Vigna lasiocarpa (Benth.) Verdc.

V. longifolia (Benth.) Verdc.

LEMNACEAE (lentilha-d'água)

Lemna aequinoctialis Welw.

L. valdiviana Phil.

Spirodela intermedia W. Koch

Wolffia brasiliensis Wedd.

W. columbiana $\mathrm{H}$. Karst.

Wolffiella lingulata (Hegelm.) Hegelm.

W. oblonga (Phil.) Hegelm.

W. welwitschii (Hegelm.) Monod

LENTIBULARIACEAE (Utriculariaceae)

Utricularia amethystina A. St.-Hil.

$U$. breviscapa Wright ex Griseb.

$U$. foliosa $\mathrm{L}$.

U. gibba $\mathrm{L}$.

$U$. hydrocarpa Vahl

U. myriocysta A. St.-Hil. \& Girard

$U$. poconensis Fromm-Trinta

$U$. simulans Pilg.

U. pusilla Vahl

U. warmingii Kamiénski (=U. hoehnei Kulm.)

LIMNOCHARITACEAE

Hydrocleys parviflora Seub.

H. nymphoides (Willd.) Buchenau

Limnocharis flava (L.) Buchenau

L. laforestii Duchass. in Griseb.

LYTHRACEAE

Cuphea melvilla Lindl. (=C. speciosa (Anders.) Kuntze Rotala mexicana Cham. \& Schltdl.

R. ramosior (L.) Koehne

$\begin{array}{lcc}\text { lagartixa } & \text { FF } & \text { AP } 6189 \\ \text { lagartixa } & \text { FF } & \text { VJP } 1788 \\ \text { camalote } & \text { E } & \text { AP } 4736 \\ & \text { E } & \text { VJP } 1688\end{array}$

erva-de-bicho E VJP 1779

lodo A $\quad$ ACC 3379

MALVACEAE

Hibiscus cisplatinus A.St.-Hil.

H. furcellatus Desv.

H. sororius L. f.

H. striatus Cav.

Malachra radiata $\mathrm{L}$.

Pavonia angustifolia Benth.

$P$. laetevirens $\mathrm{R}$. E. Fr.

P. opulifolia S. Moore

malva

algodão-bravo

A $\quad$ CDKC 766

MARANTACEAE

Thalia geniculata $\mathrm{L}$.

Thalia cf. trychocalyx M.F. Gagnep.

MAYACACEAE

Mayaca fluviatilis Aubl.

algodão-bravo

E,A $\quad$ CDKC 564

E,A AP 5637

E,A $\quad$ AP 4664

E,A VJP 3191

A,E $\quad$ AP 4730

E,A NCB 280

E,A $\quad$ AP 5082

E,A AP 3195

MELASTOMATACEAE

Acisanthera limnobios (DC.) Triana

Rhynchanthera novemnervia DC.

$\begin{array}{lcc}\begin{array}{ccc}\text { caeté } \\ \text { caeté }\end{array} & \text { E } & \text { AP 2643 } \\ \text { lodo } & \text { VJP } 3008 \\ & \text { SF,A } & \text { AP 2201 } \\ & \text { E } & \text { AP 5560 } \\ & \text { A } & \text { AP 3705 }\end{array}$


Familia/Espécie

Nome Forma Número

MENYANTHACEAE

Nymphoides indica (L.) Kuntze popular

biol. coletor

(=N. humboldtiana (Kunth) Kuntze)

N. grayana (Griseb.) Kuntze

$\begin{array}{ccc} & \text { FF } & \text { AP } 6868 \\ \text { lagartixa } & \text { FF } & \text { VJP } 2725 \\ & & \\ \text { lodo } & \text { SF } & \text { VJP } 1535 \\ \text { lodo } & \text { SF } & \text { VJP } 1414\end{array}$

Najas microcarpa $\mathrm{K}$. Schum.

$N$. guadalupensis (Spreng.) Magnus subsp. guadalupensis

NYMPHAEACEAE

Nymphaea amazonum Mart. ex Zucc.

subsp. amazonum; e N. amazonum subsp. pedersenii

camalote-da-meia-noite;

CDKC 572

largatixa; pata-de-boi FF VJP 2538

Wiersema

N. belophylla Trickett

FF

VJP 2049

$N$. gardneriana Planch.

camalote-da-meia-noite; FF

VJP 2573

N. jamesoniana Planch. largatixa

camalote-da-meia-noite; FF VJP 2564 largatixa

N. lingulata Wiersema

camalote-da-meia-noite; FF

VJP 1557 largatixa

N. oxypetala Planch.

camalote-da-meia-noite; SF,FF

VJP 1783 largatixa

N. prolifera Wiersema

camalote-da-meia-noite; FF VJP 1906 largatixa vitória-régia $\quad \mathrm{FF}$

VJP 1999

Victoria amazonica (Poepp.) Sowerby

ONAGRACEAE

Ludwigia decurrens Walt.

L. grandiflora (Michx.) Zardini (=L. uruguensis (Cambess.) Hara florzeiro

L. helminthorrhiza (Mart.) Hara (= L. natans Humb. \& Bonpl.)

L. inclinata (L.f.) P.H. Raven

lodo

L. irwinii T.P. Ramamoorthy

L. lagunae (Morong) Hara

erva-de-bicho

E VJP 1838

L. leptocarpa (Nutt.) Hara

lombrigueira

erva-de-bicho

L. nervosa (Poir.) Hara

L. octovalvis (Jacq.) P.H. Raven

FF,FL VJP 1322

L. peploides (H.B.K.) P.H. Raven

SF VJP 843

L. rigida (Miq.) Sandwith

L. sedoides (H.B.K.) Hara

L. tomentosa (Cambess.) Hara

A,E $\quad$ AP 3483

A,E AP 5398

A $\quad$ AP 1275

A,E $\quad$ AP 2153

A,E $\quad$ AP 2634

A $\quad$ VJP 769

A,E VJP 1422

FF AP 3233

A,E VJP 1042

\section{ORCHIDACEAE}

Erythrodes $\mathrm{cf}$. pumilla (Cogn.) Pabst

EP $\quad$ AP 4289

Habenaria aricaensis Hoehne

E AP 5530

Habenaria repens Nutt.

EP

AP 2756

POACEAE (Gramineae)

Acroceras zizanioides (H.B.K.) Dandy

* Brachiaria subquadripara (Trin.) Hitchc.

braquiária-do-brejo

AP 4107

tanner-grass

$\mathrm{E}$

VJP 3237

(=B. arrecta (Hack.) Stent.)

Echinochloa polystachya (H.B.K.) Hitchc.

Hymenachne amplexicaulis (Rudge) Nees

capim-de-capivara

E

VJP2714

Leersia hexandra Sw.

grameiro; arrozinho

Luziola bahiensis (Steud.) Hitchc.

L. fragilis Swallen

L. peruviana Juss. ex Gmelin

pastinho-d'água

E VJP 2713

E AP 1023

FF VJP 2737

FF VJP 2724

E AP 6845 
Familia/Espécie

POACEAE (Gramineae)

L. spruceana Benth. ex Doel.

L. subintegra Swallen

Oryza glumaepatula Steud. (=O. rufipogon Griff.)

O. latifolia Desv.

Panicum dichotomiflorum Michx. (= P. chloroticum Ness)

$P$. elephantipes Nees

P. mertensii Roth

P. rivulare Trin. (= P. pernambucense (Spreng.) Mez)

Paspalidium paludivagum (Hitchc. \& Chase) Parodi

Paspalum acuminatum Raddi

$P$. fasciculatum Willd.

$P$. repens $\mathrm{Berg}$.

P. vaginatum $\mathrm{Sw}$.

Sacciolepis myuros (Lam.) Chase

\section{POLYGONACEAE}

Polygonum acuminatum H.B.K.

P. ferrugineum Wedd.

P. hispidum H.B.K.

P. hydropiperoides Michx.

P. meissnerianum Cham. \& Schltdl.

P. punctatum Ell.

P. stelligerum Cham.

\section{PONTEDERIACEAE}

Eichhornia azurea (Sw.) Kunth

E. crassipes (Mart.) Solms

E. diversifolia (Vahl) Urb.

E. paniculata (Spreng.) Solms

Heteranthera limosa (Sw.) Willd.

H. multiflora (Griseb.) C.N. Horn

Pontederia cordata var. cordata (Muhl.) Torr.

P. cordata var. lancifolia (Muhl.) Torr.

Solms (= P. lanceolata Nutt.)

P. cordata var. ovalis (Mart. In Roem. \& Schult)

Pontederia rotundifolia L. f.

(= Reussia rotundifolia (L.f.) A. Cast.)

Pontederia subovata (Seub.) Lowden

(= R. subovata (Seub.) Solms-Laub.

Pontederia lagoensis Warm. (=R. lagoënsis

(Warm.) Solms-Laub.)

\section{POTAMOGETONACEAE}

Potamogeton pusillus L. ssp. pusillus

\section{RUBIACEAE}

Diodia kuntzei K. Schum.

D. macrophylla DC.

Pentodon pentandrus K. Schum.
A $\quad$ AP 3817
A AP 5520
A $\quad$ VJP 749

\section{SCROPHULARIACEAE}

Bacopa arenaria (J. A. Schmidt) Edwall

B. australis V. C. Souza

B. cochlearia (Huber) L. B. Sm.

B. egensis (Poepp.\& Endl.) Pennell

$\begin{array}{ll}\text { A } & \text { VJP } 1236 \\ \text { A } & \text { AP } 4760 \\ \text { A } & \text { AP } 6826 \\ \text { A } & \text { AP } 6271\end{array}$




\begin{tabular}{|c|c|c|c|}
\hline Familia/Espécie & $\begin{array}{l}\text { Nome } \\
\text { popular }\end{array}$ & $\begin{array}{c}\text { Forma } \\
\text { biol. }\end{array}$ & $\begin{array}{c}\text { Número } \\
\text { coletor }\end{array}$ \\
\hline \multicolumn{4}{|l|}{ SCROPHULARIACEAE } \\
\hline B. monnierioides (Cham.) Robinson & vick; cânfora & A & VJP 1478 \\
\hline B. myriophylloides(Benth.) Wettst. & lodo & A & VJP 429 \\
\hline B. reflexa (Benth.) Edwall & & SF & VJP 1911 \\
\hline B. rotundifolia (Michx.) Wettst. & & A & VJP 760 \\
\hline B. salzmanii (Benth.) Wettst. ex Edwall & & A & VJP 746 \\
\hline B. scabra (Benth.)Descole \& Borsini & & $\mathrm{A}, \mathrm{E}$ & AP 3268 \\
\hline B. stricta (Schrad.) Wettst. ex Edwall & & $\mathrm{E}$ & AP 4469 \\
\hline B. verticillata (Pennell \& Gleason) Pennell & & $\mathrm{E}$ & VJP 1884 \\
\hline Buchnera palustris (Aubl.) Spreng. & & A & AP 4966 \\
\hline Lindernia dubia (L.) Pennell & & A & VJP 1532 \\
\hline \multicolumn{4}{|l|}{ SOLANACEAE } \\
\hline Schwenckia angustifolia Benth. & & E & VJP 1885 \\
\hline \multicolumn{4}{|l|}{ SPHENOCLEACEAE (Campanulaceae) } \\
\hline Sphenoclea zeylanica Gaertn. & & $\mathrm{E}$ & VJP 1836 \\
\hline \multicolumn{4}{|l|}{ STERCULIACEAE } \\
\hline Byttneria genistella Triana \& Planch. & & A & AP 5508 \\
\hline B. palustris Cristóbal & & A & AP 3288 \\
\hline B. scabra Cristóbal & & A & AP 4492 \\
\hline Melochia arenosa Benth. & malva & A & AP 3356 \\
\hline M. simplex A.St.-Hil. & malva & A & AP 2626 \\
\hline \multicolumn{4}{|l|}{ TYPHACEAE } \\
\hline Typha domingensis Pers. & taboa & E & AP 4915 \\
\hline \multicolumn{4}{|l|}{ XYRIDACEAE } \\
\hline Abolboda pulchella Humb. \& Bonpl. & & A & AP 2481 \\
\hline Xyris jupicai L. C. Rich. & & E & VJP 705 \\
\hline X. savannensis Miq. & & A & VJP 264 \\
\hline
\end{tabular}

* Planta introduzida.

** Não coletada pela equipe.

Tabela 2. Número de familias, gêneros e espécies, e porcentagem de espécies, por forma biológica de macrófitas aquáticas no Pantanal (Excluindo Myriophyllum matogrosense e Ottelia brasiliensis).

\begin{tabular}{lcccc}
\hline Formas Biológicas & $\mathrm{N}^{\circ}$ de familias & $\mathrm{N}^{\circ}$ de gêneros & $\mathrm{N}^{\circ}$ de espécies & $\%$ de espécies \\
\hline emergentes & 31 & 63 & 95 & 39 \\
anfibias & 22 & 33 & 67 & 28 \\
flutuantes fixas & 11 & 14 & 28 & 11,5 \\
flutuantes livres & 9 & 12 & 19 & 8 \\
submersa fixas & 8 & 9 & 19 & 8 \\
submersas livres & 2 & 2 & 9 & 3,8 \\
epifitas & 3 & 4 & 4 & 1,7 \\
\hline
\end{tabular}

A distribuição das plantas nos corpos d'água varia de acordo com a forma biológica, profundidade da água, fatores físico-químicos e estádio sucessional. Hidrófitos geralmente têm ampla distribuição (Sculthorpe 1967), porém há espécies que ocorrem apenas em sub-regiões de solos férteis (Nabileque, Paraguai, Miranda, Abobral), p. ex.: Victoria amazonica, Eichhornia paniculata, Ceratopteris pteridoides, Nymphoides indica e Bacopa cochlearia, enquanto outras ocorrem quase somente 


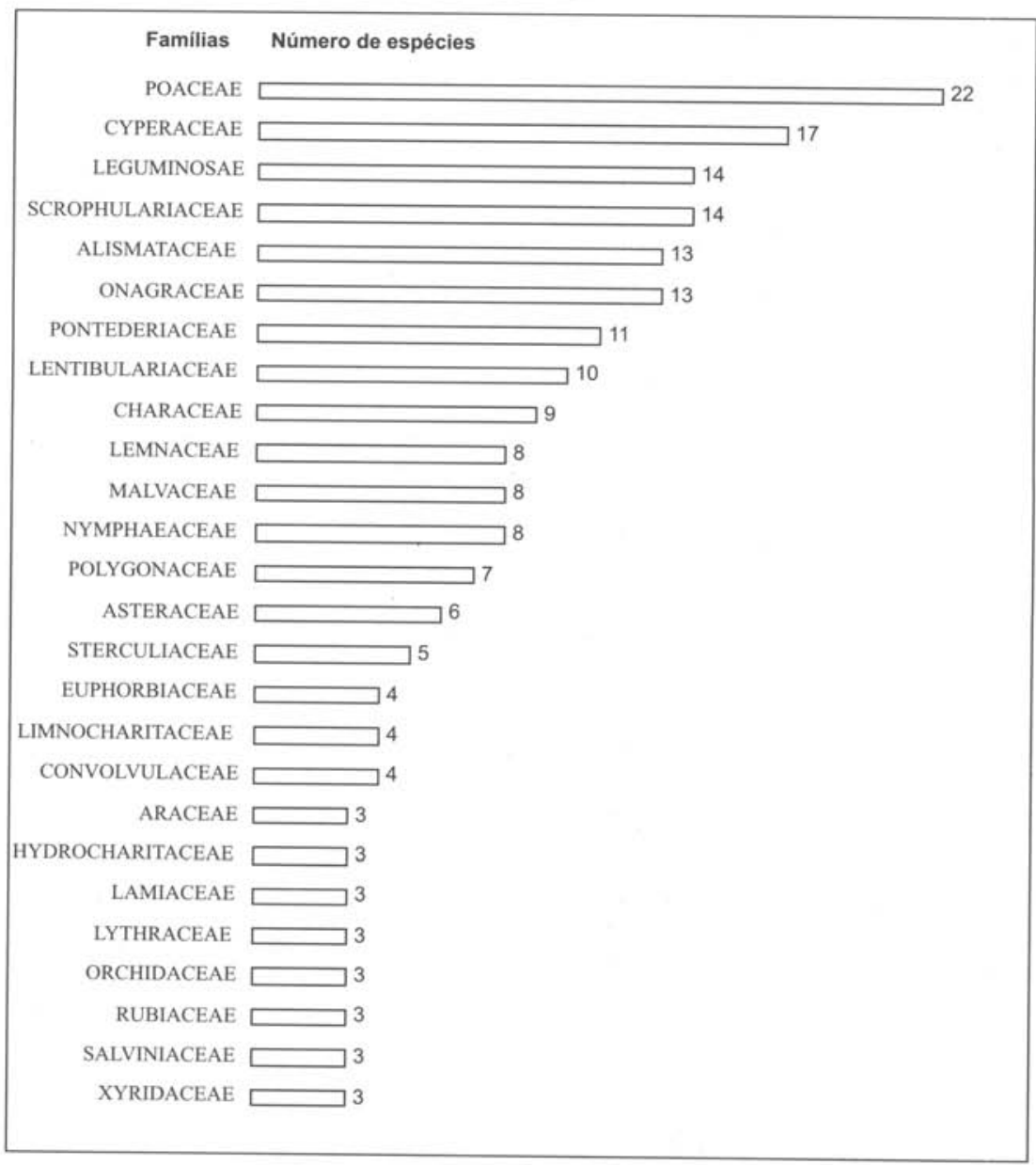

Figura 1. Distribuição do número de espécies por familia das plantas aquáticas (e de lugares úmidos), do Pantanal. Demais 28 famílias: 13 com 2 espécies e 15 com 1 espécie.

no Pantanal arenoso, como Bacopa monnierioides, Xyris savannensis e Rhynchanthera novemnervia. Brachiaria subquadripara, gramínea africana introduzida para pastagem, consta desta lista porque invade áreas alagáveis com muito vigor, eliminando outras macrófitas. Myriophyllum matogrossense é citada por Orchard (1981) para Coxipó da Ponte, Cuiabá, fora do Pantanal, porém sua ocorrência no Pantanal ainda não foi confirmada por coleta. Schessl (1997), que fez trabalho exaustivo em Poconé, não a encontrou, apenas $M$. cf. aquaticum (Vell.) Verdc., a qual ocorre no Mato Grosso e Mato Grosso do Sul, na Bacia do Alto Paraguai, fora da área do Pantanal. Prado et al. (1994) a cita, mas não foi encontrada no Herbário CH. De Ottelia brasiliensis há coleta no Herbário $\mathrm{CH}$, mas sem especificar local, provavelmente sendo de Chapada dos Guimarães (com. pess. de M. Schessl). 
Comparando a flora aquática do Pantanal com a do Rio Grande do Sul, das 331 espécies citadas no trabalho de Irgang \& Gastal (1996), 81 ocorrem no Pantanal, e das 75 famílias citadas, 52 estão representadas aqui. Lá existem famílias que são mais de clima subtropical ou temperado, como Callithricaceae, Chenopodiaceae, Juncaceae, Juncaginaceae, Podostemonaceae, Primulaceae, Ranunculaceae e Sphagnaceae, não encontradas no Pantanal.

\section{Agradecimentos}

Ao Prof. Dr. C.D.K. Cook (Universidade de Zurich) pelos ensinamentos no Curso de Plantas Aquáticas (1986). A Norma C. Bueno e Andréa da Luz Sanches, pelo companheirismo nas coletas de campo. Ao Sr. Damásio Soleto e Admar Rodrigues pela manutenção dos tanques de macrófitas aquáticas. Ao Antônio Arantes B. Sobrinho, pela dedicação no Herbário CPAP. A todas as pessoas que coletaram plantas, assim como aos especialistas de várias famílias pela identificação ou confirmação de espécies: A. Krapovickas, A. Schinini, B.E. Irgang, C.D.K. Cook, C.L. Cristóbal, E. de la Sota, E. de Melo, G. Hatschbach, J.F.M. Valls, N.C. Bueno, R. Vanni, R.J. Hickey, R.M. Harley, R.M. Lowden, T.M. Pedersen, R.R. Haynes, V.C. Souza.

\section{Referências bibliográficas}

Allem, A.C. \& Valls, J.F.M. 1987. Recursos forrageiros nativos do Pantanal Mato-grossense. Brasilia. EMBRAPA.

Bueno, N.C. 1993. Characeae do Pantanal de Mato Grosso do Sul, Brasil, Levantamento Florístico. Curitiba. Universidade Federal do Paraná. Tese de Mestrado.

Conceição, C. de A. \& Paula, J. de P. 1986. Contribuição para o conhecimento da flora do Pantanal Matogrossense e sua relação com a fauna e o homem. Anais do Simpósio De Recursos Naturais e Socio Econômicos do Pantanal, Corumbá, EMBRAPA-CPAP, UFMS, 1984. Brasília. EMBRAPA/DDT. p. 107-136. (Documentos 5).

Cook, C.D.K. 1974. Water plants of the World. The Hague. W. Junk.

Crandall-Stotler, B. 1980. Morphogenetic Designs and Theory of Bryophyte Origins and Divergence. Bio Science 30: $580-585$.

Cronquist, A. 1981. An Integrated System of classification of flowering plants. New York. Columbia University Press.

Esteves, F. A. 1988. Fundamentos de Limnologia. Rio de Janeiro. Interciência/FINEP.

Fasset, N.C. 1966. A manual of aquatic plants. Madison. University of Wisconsin.

Hamilton, S.K. 1993. Características limnológicas de importância para as plantas aquáticas no Pantanal. Resumos do II Encontro de Botânicos do Centro Oeste - CEUC/UFMS, Brasília. SBB. p. 14.

Hoehne, F.C. 1923. Phytophysionomia do Estado do Matto Grosso. Rio de Janeiro. Nacional.

Hoehne, F.C. 1948. Plantas aquáticas. São Paulo. Secretaria da Agricultura.

Irgang, B.E.; Pedralli, G. \& Waechter, J.L. 1984. Macrófitos aquáticos da Estação Ecológica do Taim, Rio Grande do Sul, Brasil. Rossléria 6: 395-404.

Irgang, B.E. \& Gastal, C.V. de S. Jr. 1996. Macrófitas aquáticas das planícies costeiras do RS. Porto Alegre. s/ed.

Martins, H.F. \& Carauta, J.P.P. 1984. Plantas aquáticas. Classificação e comentários. Atas da Sociedade Botânica do Brasil 2(13): 101-104.

Orchard, A.E. 1981. A revision of South American Myriophyllum (Haloragaceae), and its repercutions on some Australian and North American species. Brunonia 4: 27-65.

Pott, V.J. 1993. A família Lemnaceae S.F. Gray no Pantanal (Mato Grosso e Mato Grosso do Sul), Brasil. Curitiba. Universidade Federal do Paraná. Tese de Mestrado. 
Pott, V.J.; Bueno, N.C.; Pereira, R.A.C.; Salis, S.M. \& Vieira, N.L. 1989. Distribuição de macrófitas aquáticas numa lagoa da fazenda Nhumirim, Nhecolândia, Pantanal, MS. Acta Botanica Brasilica (supl.) 3(2): 153-168.

Pott, V.J.; Bueno, N.C. \& Silva, M.P. 1992. Levantamento floristico e fitossociológico de macrófitas aquáticas em lagoas da fazenda Leque, Abobral, Pantanal, MS. Anais do VIII Congresso da Sociedade Botânica de São Paulo, Campinas, 1990. Campinas. SBSP. p. 91-99.

Prado, A.L.; Heckman, C.W. \& Martins, R.F. 1994. The seasonal succession of Biotic Communities in Wetlands of the Tropical Wet-and-Dry Climatic Zone: II. The Aquatic Macrophyte Vegetation in the Pantanal of Mato Grosso, Brazil. Int. Revue ges. Hydrobiol. 79(4): 569-589.

Schessl, M. 1997. Flora und Vegetation des nördlichen Pantanal von Mato Grosso, Brasilien. Archiv naturwissenschaftlicher Dissertationen, Band 4. Wiel. Martina Galunder-Verlag.

Sculthorpe, M.A. 1967. The biology of aquatic vascular plants. London. E. Arnold.

Silva, C.J. 1984. Nota prévia sobre o significado biológico dos termos usados no Pantanal Mato Grossense. I. "Batume" e "Diquada". Revista da Universidade Federal de Mato Grosso 4: 30-36.

Silva, J. dos S.V.; Abdon, M.M. \& Silva, M.P. 1995. Delimitação do Pantanal Brasileiro e suas sub-regiões. Resumos do Encontro sobre Sensoriamento Remoto Aplicado a Estudos no Pantanal. Corumbá, MS, 1995. São José dos Campos, SP: INPE. p. 9-10.

Tur, N.M. 1965. Um caso de epifitismo aquático. Boletim da Sociedade Argentina de Botânica. 10(4): 323328.

Willis, J.C. 1973. A dictionary of the flowering plants and ferns. 8 ed. Cambridge. Cambridge University Press. 